\title{
Evaluation of Response to Preoperative Chemotherapy Versus Surgery Alone in Gastroesophageal Cancer: Tumor Resectability, Pathologic Results and Post-Operative Complications
}

\author{
Aref Kashefi Marandi ${ }^{1}$,Abolfazl Shojaiefard ${ }^{1 *}$, Ahmadreza Soroush ${ }^{1}$,Ali Ghorbani \\ Abdegah $^{1}$, Mehdi Jafari ${ }^{1}$, Mahmoud Khodadost ${ }^{2,3}$, Hossein Mahmoudzade ${ }^{1}$
}

\begin{abstract}
Gastroesophageal cancer is one of the most common types of cancer worldwide. Despite significant developments in management, 5-year survival in the developing world is less than 20 percent. Due to restricted research about the impact of preoperative chemotherapy (POC) on tumor resection, pathological response and postoperative complications in Iran, we designed and implemented the present retrospective cross- sectional study on 156 patients with gastroesophageal cancer (GEc) between 2013 and 2015 at Shariati Hospital of Tehran. Two groups were included, the first group had previously received preoperative chemotherapy and the second group had only undergone surgery. All patients were followed for at least one year after the operation in terms of tumor recurrence, relapse free survival and one-year survival. The two groups were eventually compared regarding tumor resection, pathological response, postoperative complications, recurrence rate and survival. The mean age was $66.5 \pm 7.3$ years and 78 percent were male. The tumor resectability, pathological response and postoperative complications in the group which received POC were $93.5 \%, 21.8 \%$ and $12.8 \%$, respectively, and in the surgery alone group figures for tumor resection and postoperative complications were $76 \%$ and $29.5 \%$, respectively. Also based on our study the 5-year survival in the POC group was better (79.5\% vs. $66.5 \%$ ). Using standard neoadjuvant regimens (preoperative chemotherapy/ chemoradiotherapy) beforesurgery could increase tumor resectability, pathological response, and improve the general status of the patients. Therefore using POC may be recommended over surgery alone.
\end{abstract}

Keywords: Gastroesophageal cancer - preoperative chemotherapy - tumor resection - pathological response

Asian Pac J Cancer Prev, 17, Cancer Control in Western Asia Special Issue, 231-237

\section{Introduction}

Gastroesophageal Cancer (GEc) is the eighth most common cancer in the world. The mortality rate of this cancer is estimated to exceed 407000 per year worldwide (Jemal et al., 2011). Despite outstanding advancements in managing this cancer, its 5- year survival is less than 20 percent (Dikken et al., 2012). Treating locally advanced cancers remains challenging and unsatisfactory especially when the tumor attacks the adventitia layer or the adjacent structure (T3- T4) or in cases where the adjacent lymph nodes become involved (Ajani et al., 2005; Dikken et al., 2012). A lot of effort is being made in increasing the survival of these patients. Preoperative chemotherapy (POC) based on cisplatin and fluorouracil drugs is often used before the surgery on patients suffering from gastroesophageal (GE) cancer. Although there is little evidence proving its effect on improving the prognosis, GE cancer still has a poor prognosis (Sadighi et al., 2005; Dikken et al., 2012). Numerous clinical trial studies have been conducted with the aim to examine drug doses and drug schedules (phase 1 researches), to evaluate the degree of responding to the drugs (phase 2 researches), and to evaluate the effect of a new medicine or a combination drug during a standard treating period on the overall survival in patients (phase 3 researches) in order to make progress in managing the GE cancer (Ott et al., 2011; Lee et al., 2012).

Pathologic Complete Response (PCR) is one of the initial indicators which indicates the success of preoperative chemoradiotherapy. PCR refers to the absence of live tumor cells in the microscope sample (histologic). A significant relationship has been also observed between PCR and an increase in patient survival in different meta- analyses and clinical trials (Ott et al., 2011; Valenti et al., 2011; Pera et al., 2012). Although the definitive treatment for GE cancer (and in general, gastric 
and esophageal cancer) is to surgically remove the entire tumor with clean microscopic margins, most patients still experience local and remote recurrence even after these types of surgeries (Sadighi et al., 2005; Jemal et al., 2011). All efforts are currently focused on systemic and local treatments before and after the surgery, surgery alone is not enough and the aim of prescribing the POC is to start the treatment at a time when the body tolerates the drug better and local and distant recurrences are prevented, so the disease stage decreases, the probability to increase the relapse- free survival and the total survival of the patient will therefore increase And eventually will increase the chances of successful operation (Ajani et al., 2005; Ott et al., 2011; Dikken et al., 2012; Lee et al., 2012). A number of preoperative and postoperative treatments have been examined in order to increase survival. Regarding advanced GE cancers, which include advanced local cases and metastatic diseases, the studies have revealed that palliative chemotherapy increases patient survival more than the best patient preservation treatments (Pera et al., 2012).

Many treatment protocols have been suggested in this filed which include chemotherapy protocols based on cisplatin, docetaxel, oxaliplatin, and edible fluoropyrimidines (Archie et al., 2006; Shigeoka et al., 2010; Pera et al., 2012). There is no specific treatment regimen as the gold standard and the proper regimens are the ones which are based on a combination of one of the platinum substances (cisplatin or oxaliplatin) and a fluoropyrimidine (such as FU- 5 or capecitabine). An additional selection is based on adding epirubicin or docetaxel to the above- mentioned substances (Sadighi et al., 2005; Valenti et al., 2011; Pera et al., 2012). With regard to the fact that limited number of researches have been conducted in Iran on preoperative chemotherapy, we have carried out the present research with the aim to evaluate the tumor resectability after preoperative chemotherapy, pathological response and postoperative complications, recurrence, and one- year survival of the patients.

\section{Materials and Methods}

This research conducted as a retrospectivecross-sectional and study on 156 patients between 18 to 80 years old suffering from gastroesophageal cancer (GEc) between 2013 and 2015 at Shariati Hospital of Tehran. The patients were enrolled in the research after obtaining informed consent. We designed this comparative study based on two groups, the first group of patients had previously received preoperative chemotherapy and the second group had only undergone surgery (SA). All patients were followed for at least one year after the operation in terms of tumor recurrence, relapse free survival and one- year survival. The two groups were eventually examined and compared with each other regarding tumor resection, pathological response, postoperative complications, recurrence rate and survival. The data were collected through patient records, examinations and following up the participants.

The inclusion criteria for participating in the study were as follows: the participants had to fill out the informed consent form, 18 to 80 years old, lose less than $5 \%$ weight within 3 months before participating in the study, the patients must not have experienced undergoing chemotherapy and radiation to the abdomen, presence of initial malignancies or other main associate diseases. Absence of distant metastasis including the lungs, bone, brain, and liver and ascites. The exclusion criteria were included following items : metastasis, history of sensitivity to chemotherapy drugs, infection and fever, pregnancy and breastfeeding, other malignancies within the past 5 years, not undergoing surgery after 4 weeks from the first chemotherapy, irreversible effects of preoperation chemotherapy and The patient's unwillingness to participate in the study.

All patients of the first group were received the following drug regimen: $100 \mathrm{mg}$ epirubicin (IV) and 200 mg of oxaliplatin 100 (IV) in the first day and 24- hour infusion of 400 milligrams of 5-fluoropyrimidine in days 1 through 21 . The above- mentioned regimen was repeated for all the patients in 3,21-day periods and in case of the occurrence of drug- dependent toxicity such as neutropenia, anemia, nausea and vomiting, diarrhoea, neuropathy, and etc., the drugs doses were adjusted or discontinued. Total gastrectomy surgery or esophagectomy surgery (reconstructive) was carried out along with lymphadenectomy four weeks after the last dose of preoperative chemotherapy and the resectability rate was assessed during the surgery. All the surgeries were performed simultaneously by two sufficiently- experienced surgeons in the field of gastroesophageal malignancy operations and postoperative complications such as anastomotic leaks, pneumonia, respiratory failure, surgical infection, ileus, and etc. were noted down. Checklist, examinations, pathology and radiology reports, surgeon's observations, and the information in the files of the patients were used to collect the data required by this research. After collecting the essential data, the data were analyzed with version 22 of SPSS software. The continuous variables are reported as means $\pm \mathrm{SD}$, and categorical variables are reported by number and percentage. Normality for continuous variables was determined by the Kolmogorov-Smirnov test, which revealed that the continuous variables showed normal distributions $(\mathrm{P}>0.05)$. The unpaired $t$ test was used for comparison of variables between groups. For statistical evaluation of categorical variables, we used the chi-square test and Fisher exact test as appropriate. P values of $<0.05$ were considered as significant.

\section{Results}

A total of 156 patients were examined in the present research. They were placed into two groups the first group included the patients who had received POC (100 mg epirubicin, $200 \mathrm{mg}$ of oxaliplatin, and $400 \mathrm{mg}$ of 5- FU) before the surgery and the second group included the patients who only underwent the operation. The findings demonstrated that the mean and SD of the age is $66.5 \pm$ 7.3 years. The age range of the under- study individuals was 25 to 80 years and they were classified in six groups. Most of the patients were 60- 70 years old and the minority 
Table 1. Demographic and Clinical Outcomes of the Two Groups (Distribution \& Significance)

\begin{tabular}{|c|c|c|c|c|c|c|c|}
\hline \multirow{3}{*}{$\begin{array}{l}\text { Demographic and } \\
\text { Characteristic }\end{array}$} & \multirow{3}{*}{ Levels } & \multicolumn{4}{|c|}{ Treatment } & \multirow{2}{*}{ Total } & \multirow{3}{*}{ P-value } \\
\hline & & \multicolumn{2}{|c|}{$\begin{array}{l}\text { Chemotherapy and Surgery } \\
\qquad(\mathrm{n}=78)\end{array}$} & \multicolumn{2}{|c|}{ Surgery Alone $(\mathrm{n}=78)$} & & \\
\hline & & No. & $\%$ & No. & $\%$ & $\%$ & \\
\hline Age (y) & Mean (SD) & $62.8(6.33)$ & & $60.3(8.1)$ & & $61.56(7.33)$ & $\mathrm{P}>0.05$ \\
\hline \multirow[t]{2}{*}{$\operatorname{sex}$} & male & 58.0 & 74.4 & 65.0 & 83.3 & 78.8 & 0.17 \\
\hline & female & 20.0 & 25.6 & 13.0 & 16.7 & 21.2 & \\
\hline \multirow{3}{*}{$\begin{array}{l}\text { WHO performance } \\
\text { status }\end{array}$} & 1.0 & 16.0 & 52.6 & 5.0 & 6.4 & 13.5 & 0.03 \\
\hline & 2.0 & 41.0 & 20.5 & 47.0 & 60.3 & 56.4 & \\
\hline & $>2.0$ & 21.0 & 26.9 & 26.0 & 33.3 & 30.1 & \\
\hline \multirow[t]{3}{*}{ Histologic subtype } & Intestinal & 19.0 & 24.4 & 18.0 & 23.1 & 23.7 & 0.14 \\
\hline & $\begin{array}{l}\text { Nonintestinal } \\
\text { (diffuse) }\end{array}$ & 40.0 & 51.2 & 48.0 & 61.5 & 56.4 & \\
\hline & Scc & 12.0 & 24.4 & 12.0 & 15.4 & 19.9 & \\
\hline \multirow[t]{3}{*}{ Differentiation of tumor } & Poor & 25.0 & 32.1 & 30.0 & 38.5 & 35.3 & 0.70 \\
\hline & Moderate & 42.0 & 53.8 & 38.0 & 48.7 & 51.3 & \\
\hline & Well & 11.0 & 14.1 & 10.0 & 12.8 & 13.5 & \\
\hline \multirow{3}{*}{$\begin{array}{l}\text { T category across } \\
\text { different staging } \\
\text { methods }\end{array}$} & $\mathrm{T} 2$ & 3.0 & 3.8 & 2.0 & 2.6 & 3.2 & 0.63 \\
\hline & $\mathrm{T} 3$ & 62.0 & 79.5 & 58.0 & 74.4 & 76.9 & \\
\hline & $\mathrm{T} 4$ & 13.0 & 16.7 & 18.0 & 23.1 & 19.9 & \\
\hline \multirow{4}{*}{$\begin{array}{l}\mathrm{N} \text { category across } \\
\text { different staging } \\
\text { methods }\end{array}$} & N0 & 7.0 & 9.0 & 22.0 & 28.2 & 18.6 & $<0.01$ \\
\hline & N1 & 35.0 & 44.9 & 19.0 & 24.4 & 34.6 & \\
\hline & N2 & 33.0 & 42.3 & 37.0 & 47.4 & 44.9 & \\
\hline & N3 & 3.0 & 3.8 & 0.0 & 0.0 & 1.9 & \\
\hline \multirow{3}{*}{$\begin{array}{l}\text { M category } \\
\text { across different staging } \\
\text { methods }\end{array}$} & M0 & 53.0 & 67.9 & 60.0 & 76.9 & 72.4 & 0.01 \\
\hline & M1 & 17.0 & 21.8 & 18.0 & 23.1 & 22.4 & \\
\hline & Missing & 8.0 & 10.3 & 0.0 & 0.0 & 5.1 & \\
\hline
\end{tabular}

were 30 to 40 years old. Also 123 under- study individuals were male $(78.2 \%)$ and 33 of them were female $(21.2$ $\%$ ) (Table 1).

Based on the findings of the present research, a significant relationship was seen between receiving POC and tumor resection in such a manner that almost $74 \%$ of the patients of the first group had a resectable tumor while only $63 \%$ of the patients of the second group had resectable tumors that was statistically significant $(\mathrm{P}<$ $0.005)$. Of 78 patients who had received Neoadjuvant, $36(46.2 \%)$ individuals had a pathologic relative response (G1), 32.1\% had a pathologic incomplete response (G2), and only $21.8 \%$ of the patients had a pathologic complete response (G3) (Table 2). postoperative complications have a different distribution in the two groups in such a manner that ileus, anastomotic leaks, pneumonia, and peritonitis (infection) and bleeding were not significantly different in the two groups $(\mathrm{P}>0.05)$ the two groups were however significantly different regarding wound dehiscence $(\mathrm{P}<0.08$ in first group vs. $\mathrm{P}<0.02$ in second group), also seroma formation was not reported in any of the groups. Comparing the tumor- staging findings before Neoadjuvant therapy and after the surgery indicates that preoperative chemotherapy has a significant impact on reducing the tumor stage $(\mathrm{P}<0.01)$. Based on the opinion of the surgeon the tumor recurrence was approximately $31.1 \%$ in the group which had received Neoadjuvant. Tumor recurrence was however almost $57.8 \%$ in patients who had only undergone the surgery which is statistically significant $(\mathrm{P}<0.05)$. Also a significant difference was seen between the two groups regarding the time without disease and the one- year survival values $(\mathrm{P}<0.01$ and 
Table 2. Other Characteristics of Patients According to Treatment Assignment

\begin{tabular}{|c|c|c|c|c|c|c|c|}
\hline \multirow{3}{*}{ Clinical findings } & \multirow{3}{*}{ Levels } & \multicolumn{4}{|c|}{ Treatment } & \multirow{3}{*}{$\begin{array}{c}\text { Total } \\
\%\end{array}$} & \multirow{3}{*}{ P-value } \\
\hline & & \multicolumn{2}{|c|}{ Chemotherapy and Surgery $(n=72)$} & \multicolumn{2}{|c|}{ Surgery Alone $(n=72)$} & & \\
\hline & & No. & $\%$ & No. & $\%$ & & \\
\hline \multirow[t]{2}{*}{ Resection } & R0 & 5.0 & 6.4 & 17.0 & 21.8 & 14.1 & $<0.01$ \\
\hline & $\mathrm{R} 1$ & 73.0 & 93.6 & 61.0 & 78.2 & 85.9 & \\
\hline \multirow{3}{*}{$\begin{array}{l}\text { Pathological response to } \\
\text { neoadjuant }\end{array}$} & $\mathrm{G} 0 *$ & 17.0 & 21.8 & - & - & 21.8 & - \\
\hline & G1 & 36.0 & 46.2 & - & - & 46.2 & \\
\hline & G2 & 25.0 & 32.1 & - & - & 32.1 & \\
\hline \multirow[t]{2}{*}{ Recurrence } & Yes & 24.0 & 31.1 & 44.0 & 57.8 & 44.4 & 0.05 \\
\hline & No & 53.0 & 68.9 & 32.0 & 42.2 & 55.6 & \\
\hline \multirow[t]{2}{*}{ Time without disease } & Mean $( \pm \mathrm{sd})$ & $17.5(0.6)$ & & $13.9(0.6)$ & & & $<0.01$ \\
\hline & month & & & & & & \\
\hline \multirow[t]{2}{*}{ Operating time } & Mean $( \pm$ sd) & $3.8(0.6)$ & & $3.8(0.5)$ & & & 0.89 \\
\hline & hour & & & & & & \\
\hline \multirow[t]{3}{*}{ The one-year survival } & Live & 62.0 & 79.5 & 52.0 & 66.7 & 73.1 & 0.03 \\
\hline & Died & 16.0 & 20.5 & 26.0 & 33.3 & 26.9 & \\
\hline & Missing & 8.0 & 10.3 & 0.0 & 0.0 & 5.1 & \\
\hline
\end{tabular}

Table 3. Summary of Pathological Findings

\begin{tabular}{|c|c|c|c|c|c|c|c|}
\hline \multirow{3}{*}{$\begin{array}{l}\text { Pathological find- } \\
\text { ings }\end{array}$} & \multirow{3}{*}{ Levels } & \multicolumn{4}{|c|}{ Treatment } & \multirow{2}{*}{\multicolumn{2}{|c|}{ Total }} \\
\hline & & \multicolumn{2}{|c|}{$\begin{array}{l}\text { Chemotherapy and Surgery } \\
\qquad(\mathrm{n}=78)\end{array}$} & \multicolumn{2}{|c|}{ Surgery Alone $(\mathrm{n}=78)$} & & \\
\hline & & No. & $\%$ & No. & $\%$ & No. & $\%$ \\
\hline \multirow[t]{3}{*}{ Tumor location } & Cardia & 39.0 & 50.0 & 43.0 & 55.1 & 82.0 & 52.6 \\
\hline & Distal of esophagus & 15.0 & 19.2 & 10.0 & 12.8 & 25.0 & 16.0 \\
\hline & GEj & 24.0 & 30.8 & 25.0 & 32.1 & 49.0 & 31.4 \\
\hline \multirow[t]{3}{*}{ degree } & 11 & 11.0 & 14.1 & 26.0 & 33.3 & 37.0 & 23.7 \\
\hline & 111 & 65.0 & 83.3 & 49.0 & 62.8 & 114.0 & 73.1 \\
\hline & missing & 2.0 & 2.6 & 3.0 & 3.8 & 5.0 & 3.2 \\
\hline \multirow[t]{2}{*}{ Resectin margin } & $\mathrm{R} 0$ & 66.0 & 84.6 & 57.0 & 73.1 & 123.0 & 78.8 \\
\hline & $\mathrm{R} 1$ & 12.0 & 15.4 & 21.0 & 26.9 & 33.0 & 21.2 \\
\hline \multirow{2}{*}{$\begin{array}{l}\text { Lymphnode } \\
\text { ivvolved }\end{array}$} & $\mathrm{N}+$ & 30.0 & 38.5 & 60.0 & 76.9 & 90.0 & 57.7 \\
\hline & N- & 48.0 & 61.5 & 18.0 & 23.1 & 66.0 & 42.3 \\
\hline
\end{tabular}

$\mathrm{P}<0.03$, respectively) based on the findings of our study (Table 3).

\section{Discussion}

Although the definitive treatment for GE cancer (and in general, gastric and esophageal cancer) is to surgically remove the entire tumor with clean microscopic margins, most patients still experience local and distant recurrence even after operation. Today, Different researches have clearly demonstrated that surgery alone is not enough and all efforts are currently focused on systemic and local treatments before and after the surgery, the aim of prescribing $\mathrm{POC}$ is to start the treatment at a time when the body tolerates the drug better and local and distant recurrences are prevented and the possibility that the surgery will succeed increases. Different researches with numerous treatment regimens have been conducted on the effect of POC on tumor resectability, pathological response, postoperative complications, tumor recurrence, and patient survival which have resulted more or less similar outcomes. In contrast, there are researches which have not obtained good results regarding using POC (Flamant Y, 1999; Cunningham et al., 2006). It was seen based on our findings that there is a significant relationship between receiving POC and tumor resectability. 73 individuals $(93.5 \%)$ of the total 78 people who had received POC were resectable while 
only 60 (76\%) individuals in the second group $(\mathrm{P}<0.01)$ were resectable. The results of a clinical trial study with (paclitaxel/ $5 \mathrm{w}$ ) treatment regimen indicated that the tumor had completely resected in 92 percent of the patients of POC group which was significantly different from the $69 \%$ in the surgery alone group $(\mathrm{P}<0.01)$. In contrast to this study, another RCT study in America showed that the two groups are almost similar in terms of tumor resection. But the tumor was however significantly smaller in the group that had received POC and fewer cases of advancement were seen in them (Urba et al., 2001; Ando et al., 2003).

Postoperative complication distribution was different in the patients. The complications were generally less frequent in the POC group in comparison to the surgery alone group (14 cases vs 19 cases). Ileus, anastomotic leaks, pneumonia, and peritonitis (infection) and bleeding were not significantly different in the two groups $(\mathrm{P}>0.05)$. There was however a significant statistical difference regarding wound dehiscence $(\mathrm{P}<0.03)$. The most frequent reported complications was anastomotic leak with 10 cases which included 30 percent of all the complications it is also worth mentioning that seroma was not formed in any of the patients. In a retrospective trial study with a combination treatment regimen of cisplatin and FU-5, comparing the results of complications occurring during the operation and postoperative complications indicated that only bleeding during the surgery and anastomotic leaks were significantly different in the two groups $(\mathrm{P}<$ $0.05)$ all the other matters were not significantly different. Based on the findings of this study, the surgery duration $(\mathrm{P}=0.983)$, blood loss during the operation $(\mathrm{P}=0.84)$, and tumor differentiation from the neighbouring tissues were not significantly different which is similar to the results of the studies which were conducted in this field (Cunningham et al., 2006; Shigeoka et al., 2010; Ychou et al., 2011).

$36(46.2 \%)$ individuals from amongst the total of 78 patients in our study who had received POC had a relative pathological response, $32.1 \%$ had an incomplete pathological response, and $21.8 \%$ of the patients had a pathologic complete response (PCR). PCR is considered the absence of the remains of the tumor in surgical samples which is one of the factors indicating the success of the surgery. The researches of other researchers resulted in similar findings which were consistent with our study. One of these researches was a second- phase trial study which used (oxaliplatin, cisplatin, and 5-FU) treatment regimen for 6 to 8 weeks. The result indicated that PCR was seen in 25 percent of the gastroesophageal junction cancer cases (Cunningham et al., 2006). Another research demonstrated that PCR was seen in $33 \%$ of the patients who were being treated with POC which was similar to the results obtained from Khaldoun Almhanna et al.'s research in Florida (25\%). In Simon's study however, the PCR to chemotherapy was $58 \%$ while only $6.7 \%$ of the patients were affected by PCR (Kelsen et al., 1998; Urba et al., 2001; Ychou et al., 2011).

Also comparing the surgery margin pathology (SMP) frequency distribution (the presence of tumor cells in the margin of the removed tissue) in the two under- study groups indicates that no tumor cells were seen in the margin of the removed tissue (R0) in almost $85 \%$ of the patients in the POC group while this was true for almost $73 \%$ of the patients who underwent surgery alone $(\mathrm{P}<$ 0.058). In a retrospective clinical trial study, no tumor cells were seen in the margin of the removed tissues in 94 percent ( $\mathrm{R} 0$ ) of the patients who had received Cisplatin which was better than our study. It is also worth mentioning that based on the findings of this study and comparing them with the findings of tumor staging before POC and after surgery, chemotherapy has had a significant impact in reducing the tumor stage $(\mathrm{P}<0.01)$ (Ando et al., 2003; Allum et al., 2009).

Based on the opinion of the surgeon, tumor recurrence was seen in almost $31.1 \%$ of the POC group but it was $57.8 \%$ in the second group $(\mathrm{P}<0.05)$. Tumor recurrence was different in studies conducted in different countries and it seems like due to the different in the target population and the cells involved, the pathological response to Neoadjuvant depends on the expertise of the surgeon and etc. most of the researches which have been conducted in this field approve that using a standard treatment regimen significantly increases the possibility of tumor recurrence. For example a clinical trial research reported the tumor recurrence in the POC group and the surgery alone group 29 and 50\%, respectively $(\mathrm{P}<0.01)$. Some other studies however claimed that although using POC decreases tumor recurrence in short- term, it does not have a noticeable and significant effect of distant recurrence of the tumor (Flamant Y, 1999; Urba et al., 2001; Weber et al., 2001). It has also been reported in different studies that in addition to decreasing the possibility of tumor recurrence in comparison with surgery alone group, the use of POC significantly increases the disease- free time or the time period between the surgery and tumor recurrence. This was also obtained in our study in such a manner that the mean disease- free time was 17.45 months in the POC group and 13.92 months in the surgery alone group which was statistically significant $(\mathrm{P}<0.01)$. It must also be noted that based on our study, the patients who had been treated with POC needed lesser postoperative chemotherapy $(\mathrm{P}<0.04)$.

Based on the findings of this research the one- year survival of the patients in the first group was greater than that of the second group. The one- year survival of the patients of the first group was $79.5 \%$ and $66.5 \%$ in the second group $(\mathrm{P}<0.03)$. On the basis of the researches conducted in this field, including an RCT study which demonstrated that the five- year survival of the patients in the POC group was $36 \%$ versus $23 \%(\mathrm{P}<0.01)$ (Law et al., 1997; Weber et al., 2001), the results of John Urschel's meta- analysis (Urschel and Vasan, 2003) (consisting of 9 randomized clinical trials: 116 patients) indicates that the one- year, two- year, and three- year survival of the preoperative chemotherapy group was 79, 77, and 66 percent, respectively, which was similar with our study. Moreover, based on the results obtained from this study the survival of the patients in the groups which had used a combination of POC and chemoradiotherapy was estimated to be greater than the group which had 
been treated with chemotherapy or radiotherapy alone. However, despite all the numerous studies which have been carried out about the outstanding effect of using POC to increase the patient survival chances, there are a few studies which state that chemotherapy and chemoradiotherapy do not affect the total survival rate of the patients and they only prolong the postoperative disease- free time and decrease the mortality rates caused by cancer (Working, 2002; Berger et al., 2005; Gebski et al., 2007).

The performance status of the patients was evaluated in both groups in this study based on WHO performance scale (0-5). The performance status of the patients was better in the first group after the surgery in comparison with the second group $(\mathrm{P}<0.04)$. This variable is of importance since approximately half of the patients suffering from GE cancer do not have the physical conditions to undergo the surgical procedure. In other words, they are unable to tolerate the surgery and 20-30 percent of the patients who are in better conditions will be affected by respiratory problems and 10-15 percent will have major heart problems which eventually leads to losing the patient. Therefore adopting proper protocols for using Neoadjuvant before the surgery could improve the general status of the patient, decrease the disease's recurrence changes or delay it, decrease mortality and increase patient survival rate.

Based on the findings of the present research and the other researchers conducted in this field, using standard regimens of Neoadjuvant (preoperative chemotherapy/ chemoradiotherapy) before the operation could increase tumor resectability, increase the pathological response, and improve the general status of the patients, therefore using POC is preferred to surgery alone.

\section{Acknowledgements}

The authors certify that no other persons have made substantial contributions to this manuscript. No conflict of interest exists in relation to the submitted manuscript. Also, this article was an independent research with no financial aid.

\section{References}

Ajani JA, Mansfield PF, Crane CH, et al (2005). Paclitaxel-based chemoradiotherapy in localized gastric carcinoma: degree of pathologic response and not clinical parameters dictated patient outcome. J Clin Oncol, 23, 1237-44.

Allum WH, Stenning SP, Bancewicz J, et al (2009). Long-term results of a randomized trial of surgery with or without preoperative chemotherapy in esophageal cancer. $J$ Clin Oncol, 27, 5062-67.

Ando N, Iizuka T, Ide H, et al (2003). Surgery plus chemotherapy compared with surgery alone for localized squamous cell carcinoma of the thoracic esophagus: a Japan Clinical Oncology Group Study-JCOG9204. J Clin Oncol, 21, 4592-96.

Archie V, Kauh J, Jones DV, et al (2006). Gastric cancer: standards for the 21st century. Crit Rev Oncol Hematol, 57, 123-31.

Berger AC, Farma J, Scott WJ, et al (2005). Complete response to neoadjuvant chemoradiotherapy in esophageal carcinoma is associated with significantly improved survival. $J$ Clin Oncol, 23, 4330-37.

Cunningham D, Allum WH, Stenning SP, et al (2006). Perioperative chemotherapy versus surgery alone for resectable gastroesophageal cancer. $N$ Engl J Med, 355, 11-20.

Dikken JL, van de Velde CJ, Coit DG, et al (2012). Treatment of resectable gastric cancer. Therap Adv Gastroenterol, 5, 49-69.

Flamant Y CJ, Huguier M, Quandalle P, et al (1999). Chimiothérapie adjuvante après résection des adénocarcinomes gastriques: étude prospective contrôlée de l'effet de l'association 5FU, acide folinique et cisplatine. Gastroenterol Clin Biol, 21, 16.

Gebski V, Burmeister B, Smithers BM, et al (2007). Survival benefits from neoadjuvant chemoradiotherapy or chemotherapy in oesophageal carcinoma: a meta-analysis. Lancet Oncol, 8, 226-34.

Information on the Dutch CRITICS trial [Online]. Available: http://clinicaltrials.gov/ct2/show/NCT00407186 [Accessed 14 December 2010].

Jemal A, Bray F, Center MM, et al (2011). Global cancer statistics. CA Cancer J Clin, 61, 69-90.

Kelsen DP, Ginsberg R, Pajak TF, et al (1998). Chemotherapy followed by surgery compared with surgery alone for localized esophageal cancer. $N$ Engl J Med, 339, 1979-84.

Law S, Fok M, Chow S, et al (1997). Preoperative chemotherapy versus surgical therapy alone for squamous cell carcinoma of the esophagus: a prospective randomized trial. J Thorac Cardiovasc Surg, 114, 210-7.

Lee DJ, Sohn TS, Lim DH, et al (2012). Phase I study of neoadjuvant chemoradiotherapy with S-1 and oxaliplatin in patients with locally advanced gastric cancer. Cancer Chemother Pharmacol, 69, 1333-38.

Ott K, Lordick F, Blank S, et al (2011). Gastric cancer: surgery in 2011. Langenbecks Arch Surg, 396, 743-58.

Pera M, Gallego R, Montagut C, et al (2012). Phase II trial of preoperative chemoradiotherapy with oxaliplatin, cisplatin, and 5-FU in locally advanced esophageal and gastric cancer. Ann Oncol, 23, 664-70.

Sadighi S, Raafat J, Mohagheghi M, et al (2005). Gastric carcinoma: 5 year experience of a single institute. Asian Pac J Cancer Prev, 6, 195-6.

Shigeoka H, Imamoto H, Nishimura Y, et al (2010). Complete response to preoperative chemoradiotherapy in highly advanced gastric adenocarcinoma. World J Gastrointest Oncol, 2, 282-6.

Urba SG, Orringer MB, Turrisi A, et al (2001). Randomized trial of preoperative chemoradiation versus surgery alone in patients with locoregional esophageal carcinoma. J Clin Oncol, 19, 305-13.

Urschel JD, Vasan H (2003). A meta-analysis of randomized controlled trials that compared neoadjuvant chemoradiation and surgery to surgery alone for resectable esophageal cancer. Am J Surg, 185, 538-43.

Valenti V, Hernandez-Lizoain JL, Beorlegui MC, et al (2011). Morbidity, mortality, and pathological response in patients with gastric cancer preoperatively treated with chemotherapy or chemoradiotherapy. J Surg Oncol, 104, 124-9.

Weber WA, Ott K, Becker K, et al (2001). Prediction of response to preoperative chemotherapy in adenocarcinomas of the esophagogastric junction by metabolic imaging. $J$ Clin Oncol, 19, 3058-65.

Working MRCOC (2002). Surgical resection with or without preoperative chemotherapy in oesophageal cancer: a randomised controlled trial. Lancet, 359, 1727-33. 
Ychou M, Boige V, Pignon J-P, et al (2011). Perioperative chemotherapy compared with surgery alone for resectable gastroesophageal adenocarcinoma: an FNCLCC and FFCD multicenter phase III trial. $J$ Clin Oncol, 29, 1715-21. 